Available online @ https://jiem.jnnce.ac.in https:www.doi.org/10.37314/JJEM.2021.050105 Indexed in International Scientific Indexing (ISI) Impact factor: 1.025 for 2018-19

Published on: 30 September 2021

\title{
ZigBee Wireless Communication Technology
}

\author{
Rashmi M Hullamani ${ }^{1}$, Kavya T $\mathbf{N}^{2}$ \\ ${ }^{1,2}$ Department of Electronics and Telecommunication Engineering, \\ JNN College of Engineering, Shimoga, Karnataka, India \\ rashmimh@jnnce.ac.in, kavyagirija1999@gmail.com
}

\begin{abstract}
ZigBee Technology is a wireless communication technology. Wireless Communication is not a new technology as we already have short range communication standards, like Wi-Fi and Bluetooth. But ZigBee is specially built for control and Sensor networks. Other wireless technologies are not quite suitable for this specific application. ZigBee is a wireless technology standard that defines a set of communication protocols for short range communications. This paper gives an overview of the ZigBee Technology and it covers ZigBee working, applications and some of general characteristics of ZigBee standard.
\end{abstract}

Keywords: ZigBee, IEEE 802.15.4, Home automation, Medical Data Collection.

\section{Introduction}

IEEE 802.15.4 or ZigBee is a standard that addresses the need of very low-cost implementation of low power devices with low data rate for short range wireless communications. ZigBee Figure 1 is of the most commonly used standard for Internet of Things (IOT). It is also an open source standard that was developed by ZigBee Alliance Which is an organization created in 2002 [1], [2] [7].

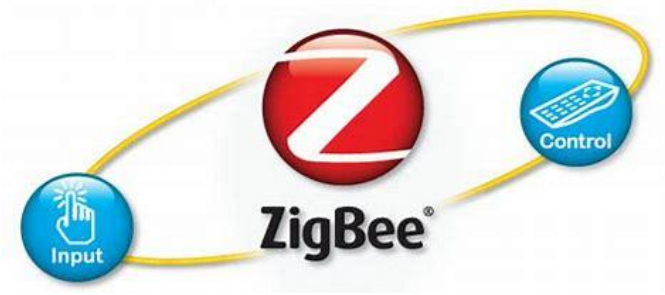

Figure 1: ZigBee

ZigBee defines three types of devices:

1. Coordinator

2. Routers

3. End devices
1) Coordinator: ZigBee Coordinator is the most capable device, it is the device that starts the network originally, so it is the root of the network and there is exactly one coordinator device in each network. The coordinator performs many tasks like channel selection, before coordinator stars the network it should perform channel scan to select the good channel that is with channel with least interferences to be used for the network, it also assigns a unique ID to the network and it also allocates unique address to each device so anytime device [2] joins the network, coordinator initiates and transfers messages in the network. Coordinator network is as shown in the below Figure. 2.

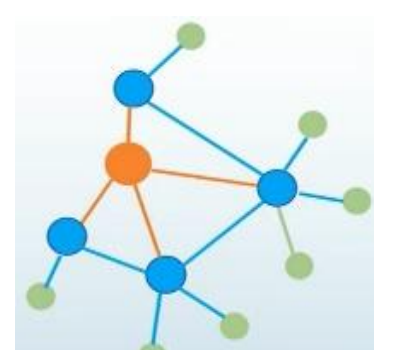

Figure 2: Coordinator 
2) Routers: Router act intermediate nodes between the coordinator and the end devices, they are responsible for route traffic between different nodes, they also receive and store messages intended for their children, at certain occasions routers can allow other routers end devices to join the network. Routers are indicated in the Figure 3.

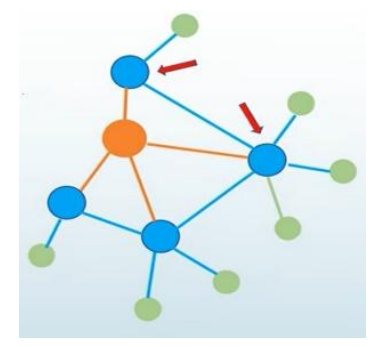

Figure 3: Routers

3) End devices: End devices contain just enough information to talk to the parent node. They may sleep (a standby), which makes end devices a suitable choice for battery operated devices. All traffic to an end device is first routed to its parent. The end device is responsible for requesting any pending messages from its parent. They are represented by A and B in the below Figure 4 .

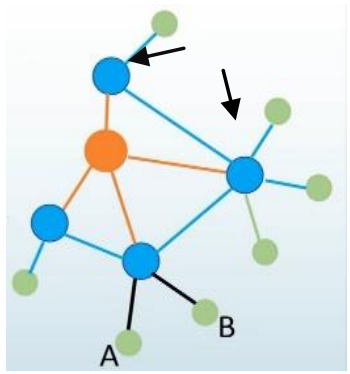

Figure 4: End devices

There are three types of network topologies that ZigBee supports

- Star Topology

- Mesh Topology

- Tree Topology

- Star Topology: This is the simplest and less expensive to implement and that is the advantage of this topology. There are no routers in this architecture. Hence end device cannot communicate directly with another end device. Star Topology is as shown in the below Figure 5.

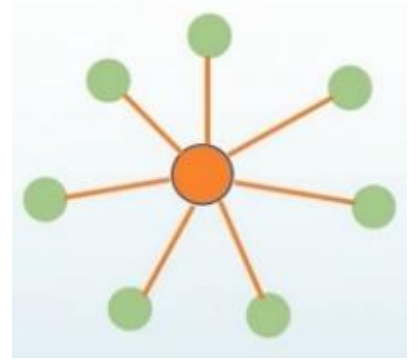

Figure 5: Star Topology

- Some of the drawbacks of this topology is that if the coordinator fails then the whole network fails, also the range of the network is limited to the range of the coordinator itself.

- ZigBee is also used in Medical Data collection; this application can be implemented by Star Topology.

- Health Monitoring System using Star Topology is illustrated in Figure 6 [3].

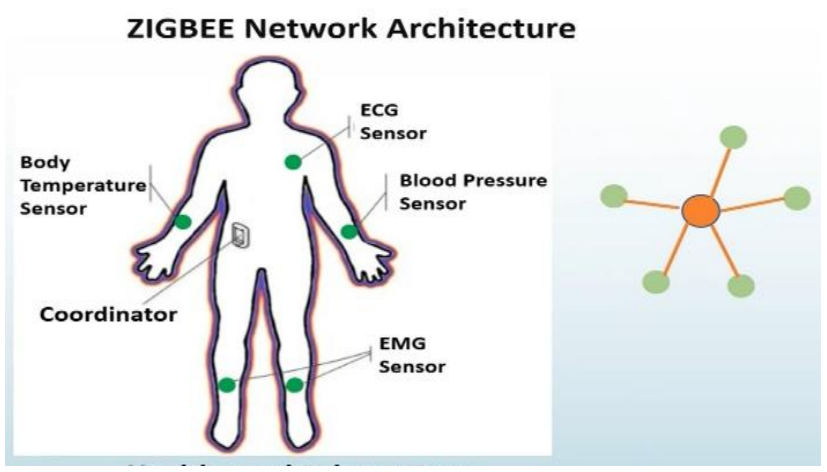

Figure 6: Health Monitoring System using Star Topology

- Mesh Topology: In the mesh network every node is connected with the neighbouring node (except for the end devices).

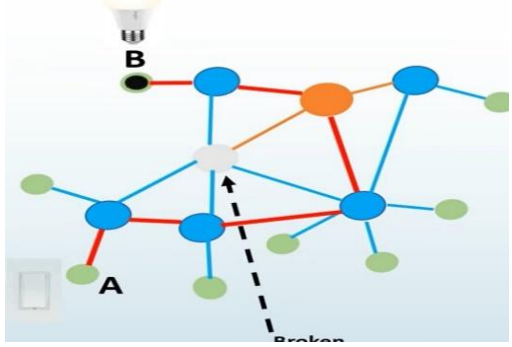

Figure 7: Mesh Topology 
- A message hops from one device to another in order to reach its destination, one of the advantage of this topology is, if a node fails, data can be re-routed using another path this is called self-Healing process in Mesh Network. Illustration of mesh network can be seen in Figure 7.

- Mesh Network Topology is used in Home Automation, The Home Automation can always add more devices, if we have 4 to 6 devices then Star Topology is enough, but as more devices are being added then Mesh Topology is used. Figure 8 show the Mesh Network implemented in Home Automation [4].

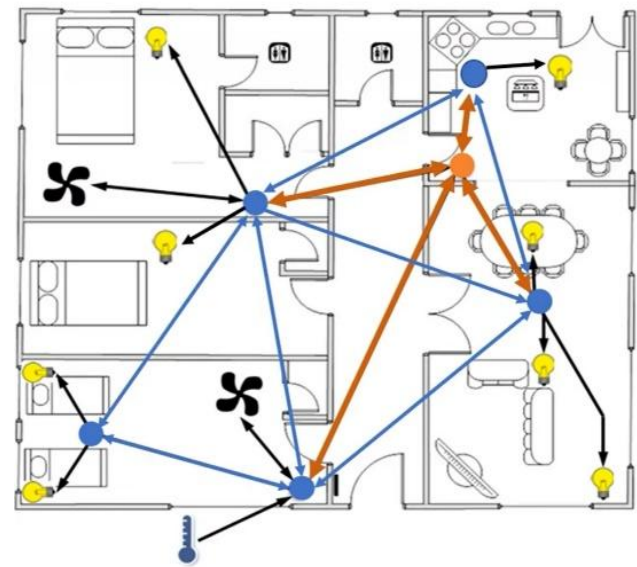

Figure 8: Mesh Network implemented in Home Automation

- Tree Topology: This Topology is not so different from Mesh configuration, except that routers are not interconnected.

- Cluster Tree Network: This Network let the routers to be connected to expand network range of the network. It is shown in Figure 9.

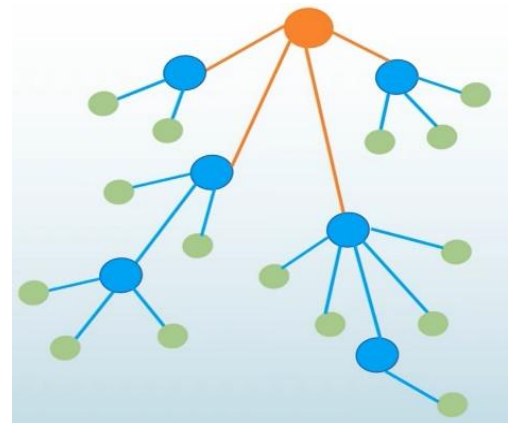

Figure 9: Cluster Tree Topology

\section{Channel Access}

- The coordinator assigns only one channel to the network upon network establishment which means all the devices will have to share this single channel to communicate.

- There are two methods for channel access:

-Contention based method.

-Contention free method.

- Contention-free method:

The coordinator dedicates a specific time slot to each device. This is called a guarantees time slot (GTS).

- The time slots cannot overlap with each other

- A star network with five devices and a coordinator to correctly assign GTS, it is shown in the Figure 10. The coordinator needs to ensure that all the devices in the network are synchronized and to achieve this coordinator transmits periodically a message called Beacon.

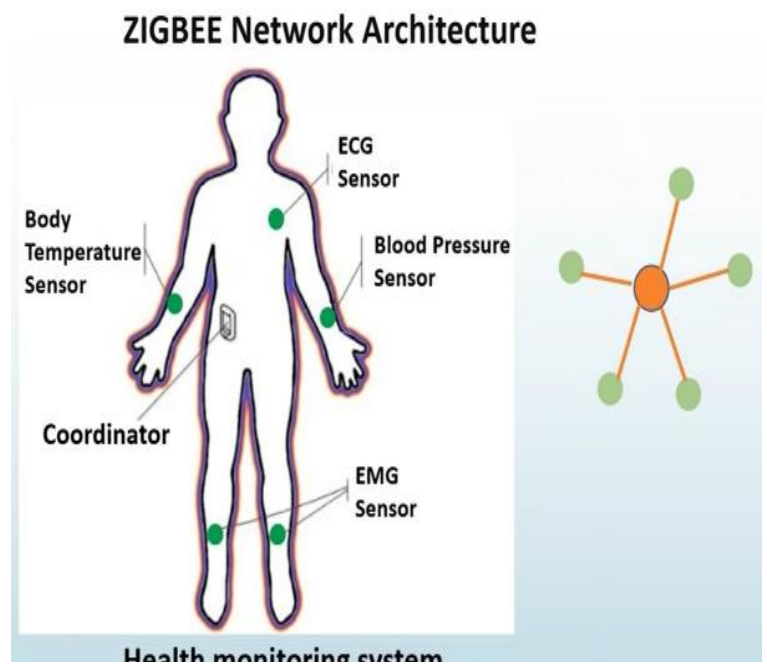

Figure 10: Coordinator assigning GTS in the star network

- Beacon: It is used to synchronize the clocks of all devices in the network [5].

- The coordinator transmits periodically a message called Beacon, once the devices receive the beacon they will adjust or sink to their clock to the coordinator clock, the reason this is accomplishing first, is because the beacon contains information on when each 
device can transmit data, so at this time all devices know ahead of time when they can transmit each device would wait for its turn and transmit its data add at that assigned timed slot, the coordinator transmits beacon periodically so the same operation repeats over time the time slot allocation may differ from one beacon period to another not all devices need to transmit every beacon period. This can be seen in Figure11. End device 2 and 4 are not transmitting in the second beacon period and this can be pre-programmed in the coordinator by the developer, because end devices know ahead of time when they can transmit they will go to sleep and they will only wake up to either transmit data or to listen to the beacon, this is what enables low power consumption in ZigBee standard.

- Contention based method:

- For the contention based method devices do not need to be synchronized.

- The Carrier Sense Multiple Access Collision Avoidance mechanism is used by all devices to access the channel.

- In CSMA/CA anytime a device wants to transmit:

It first goes into receive mode to detect if there is any signal in the channel and it will only transmit the data if the channel is clear. However, if the channel is not clear, the device backs off for a random period of time and tries again.

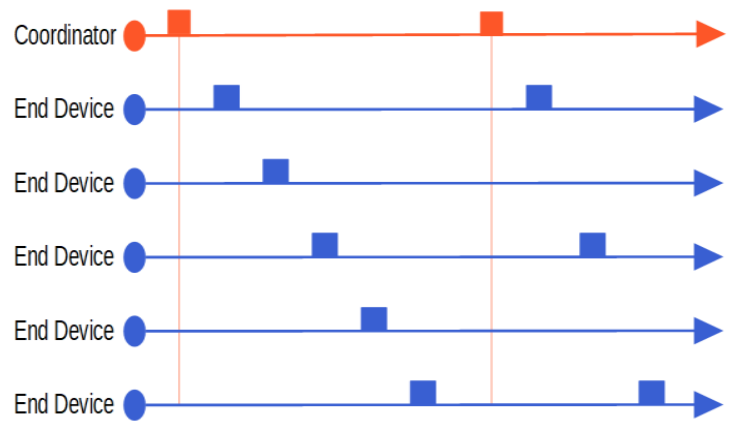

Figure 11: Contention-free method

\section{Zig Bee Applications}

The most prominent applications of ZigBee are in
1. Home automation

2. Medical data collection

3. Industrial control systems

\subsection{ZigBee in Home Automation:}

We basically have home automation controller with a software application that controls smart devices[4] [5]. The controller is also called a Central Hub or simply Hub, the Gateway is the interface between ZigBee network and the web, the gateway is connected to the internet and it will lead the home owner to control his or her smart home devices from virtually anywhere in the world.

Some of the smart home devices that ZigBee supports include

- Lights

- Door locks

- switches

- Smoke Detectors

- Fans

- Appliances

- Etc....

Connections of smart home devices in home automation are shown in the Figure 12.

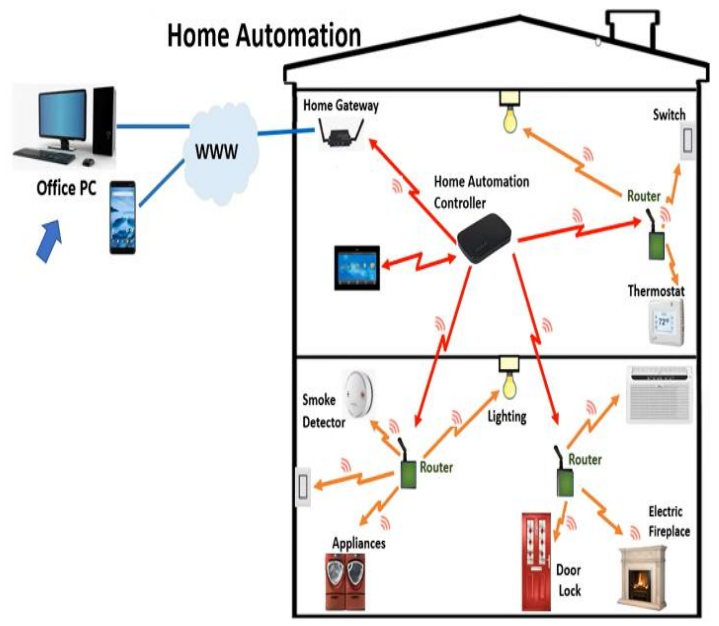

Figure 12: Home Automation

Figure 13 shows how different wall switches control different lights on the house, the wall 
switches are not connected with any electrical wire, because they are battery operated, by switching on and off any of the switches a message will be sent to the controller, which will relay that message to the appropriate light board to either turn it on or turn it off.

\section{Home Automation}

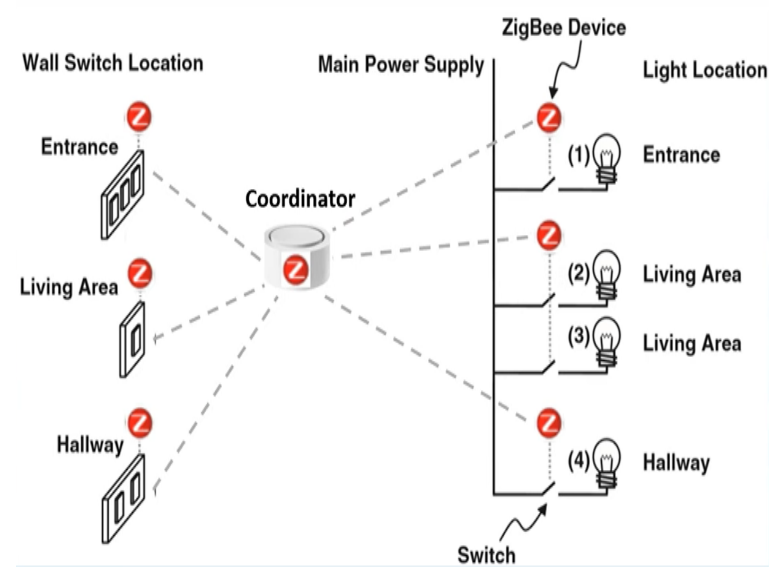

Figure 13: Working of home automation using ZigBee network

\subsection{Medical data collection}

Here a patient can be wearing ZigBee sensors that can collect information, such as blood pressure, body temperature, heart rate etc... This information will be sent through the ZigBee gateway over the internet to a personal computer in a hospital, so that a physician or a nurse can monitor the patient's health [6]. Working of Remote Monitoring System using ZigBee sensors is as shown in the Figure14.

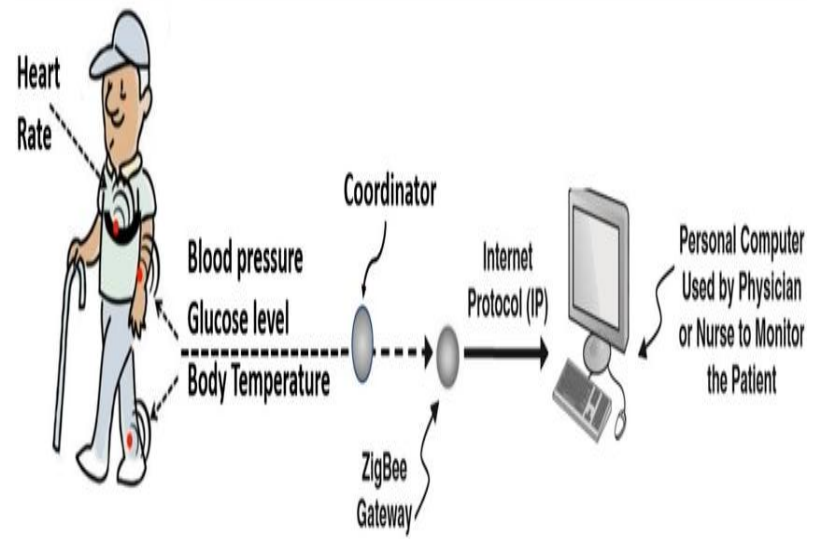

Figure 14: Remote Monitoring System using ZigBee sensors
We might think that why can't we use Wi-Fi or Bluetooth technology for these devices, the reason is that Wi-Fi uses a lot of power which is not good for battery- powered devices or devices where low power consumption is important, such as sensors, also all devices here use low data rate, while $\mathrm{Wi}-\mathrm{Fi}$ uses high data rate, so it is an overkill to use Wi-Fi in this application.

Bluetooth is also not be a good fit, because Bluetooth network can only support a maximum of seven devices in a network, while ZigBee is designed to support hundreds of devices on a network.

\section{General Characteristics of ZigBee Standard}

Some of the general characteristics of the ZigBee are

1. Low power consumption: ZigBee devices can typically operate for a several years on a single battery.

2. Low data rate: $20 \mathrm{kbps}-250 \mathrm{kbps}$

WIFI: $11 \mathrm{Mbps}$, Bluetooth: $1 \mathrm{Mbps}$

3. Short range:

Up to 75-100 meters (in indoor)

Up to $300+$ meters (line of sight)

4. Network joint time: $\sim 30$ milli sec WIFI: Up to 3 seconds,

Bluetooth: Up to 10 seconds

5. Support small and large networks:

Up to 65000 devices (in theory)/240 devices (in practical)

WIFI: Up to 32 devices,

Bluetooth: Up to 7 devices

6. Low cost of products and cheap implementation: Simplicity of the technology and it is also open source protocol. 
7. Security: ZigBee uses AES cryptographic algorithm for data encryption and data authentication.

8. This is the standard that banks are using for their online operations, it is also common place in government agencies, so it is one of the most robust and secure cryptographic algorithm.

The parameters of different technologies are presented in Table 1.

Table 1: Table Type Styles

\begin{tabular}{|c|c|c|c|}
\hline \multirow{2}{*}{ Technology } & \multicolumn{3}{|c|}{ Parameters of different technologies } \\
\hline & Frequency & $\begin{array}{c}\text { Transmission } \\
\text { Rate }\end{array}$ & Modulation \\
\hline Bluetooth & $\begin{array}{l}2.4-2483.5 \\
\mathrm{GHz}\end{array}$ & $\begin{array}{l}1 \mathrm{Mbps} \\
2 \mathrm{Mbps} \\
3 \mathrm{Mbps}\end{array}$ & $\begin{array}{l}\text { GFSK } \\
\mathrm{Pi} / 4 \\
\text { DQPSK } \\
\text { 8DPSK } \\
\end{array}$ \\
\hline ZigBee & $\begin{array}{l}2.4-2.4835 \\
\mathrm{GHz} \\
902-928 \mathrm{MHz} \\
868-868.6 \\
\mathrm{MHz}\end{array}$ & $\begin{array}{l}250 \mathrm{kbps} \\
40 \mathrm{kbps} \\
20 \mathrm{kbps}\end{array}$ & $\begin{array}{l}\text { O-QPSK } \\
\text { BPSK }\end{array}$ \\
\hline Wi-Fi & $2.4 \mathrm{GHz}$ & $11 \mathrm{Mbps}$ & QPSK \\
\hline
\end{tabular}

\section{Operating Frequency Bands}

There are three frequency bands currently assigned to ZigBee

The first frequency band is currently assigned to $\mathrm{ZigBee}$. As shown in the Figure 15 first frequency band is the $868 \mathrm{MHz}$ and it includes only one channel this band is allocated in Europe.

\section{$868 \mathrm{MHz}$ \\ Channel 0}

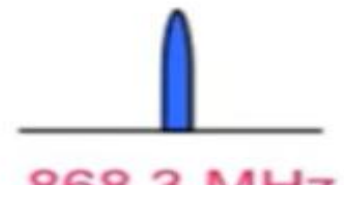

Figure 15: Frequency band allocated in Europe
The second band is the $915 \mathrm{MHz}$ and it is shown in the Figure 16 it includes ten channels and it can be found in US and Australia.

\section{$915 \mathrm{MHz}$}

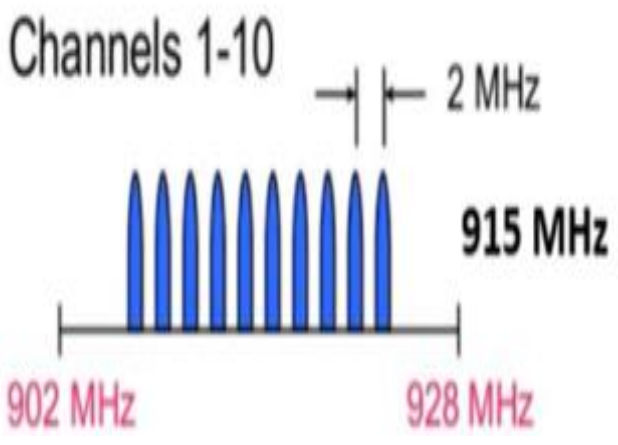

Figure 16: Frequency band allocated in US, Australia

The third band is the $2.4 \mathrm{GHz}$ band and that is available across the world [7]. The overview of Frequency bands allocated across the world is as shown in the Figure 17. Now once ZigBee network is established only one channel will be selected for that network.

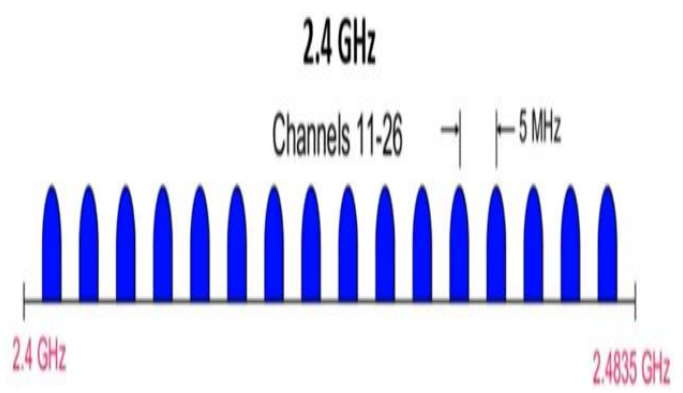

Figure 17: Frequency band allocated across the world

\section{References}

1. Zhou Yiming, Yang Xianglong, Guo Xishan, Zhou Mingang, Wang Liren. A Design of Greenhouse Monitoring \& Control System Based on ZigBee Wireless Sensor Network. Wireless Communications, Networking and Mobile Computing, 2007. WiCom 2007. International Conference.2007.P2563 - 2567.

2. Liting Cao, Wei Jiang, Zhaoli Zhang, Networked wireless meter reading system 
based on ZigBee technology. Control and Decision Conference, 2008.Chinese.2008, $3455-3460$.

3. Kwang Koog Lee,Seong Hoon Kim, Hong Seong Park.Cluster Label-based ZigBee Routing Protocol with High Scalability. Proceedings of the Second International Conference on Systems and Networks Communications (ICSNC 2007), Cap Eterel, France, 25-31 August 2007, 65-72.

4. Drew Gislason, Zigbee Wireless Networking, Elsevier Inc, Newnes, 2008.

5. Zhang Weiyong, Feng Lin, Wei Zhenchun, Research on home networking with Zigbee, Journal of Hefei University of Technology (Natural Science), Vol. 28, No. 7, July 2005, 755-799.
6. Dechuan Chen, Meifang, Wang, A Home Security Zigbee Network for Remote Monitoring Application, 2006 Proceedings of the IET International Conference on Wireless Mobile and Multimedia Networks, hangzhou, China, 6-9 November 2006,

7. Yang Junjie, LV Jian and Wei Chunjuan, A Wireless Solution for Substation Perimeter Safety Monitoring System Based on Zigbee Communication Technology, Proceedings of the International Conference On Computer Design And Applications, Vol. 4, Qinhuangdao, China, 25-26 June 2010, 520523. 